\title{
German S3 Evidence-Based Guidelines on Focal Therapy in Localized Prostate Cancer: The First Evidence-Based Guidelines on Focal Therapy
}

\author{
Angelika Borkowetz ${ }^{\mathrm{a}, \mathrm{b}, \mathrm{c}}$ Andreas Blana ${ }^{\mathrm{a}, \mathrm{b}, \mathrm{d}}$ Dirk Böhmer ${ }^{\mathrm{e}, \mathrm{f}}$ Hannes Cash ${ }^{\mathrm{b}, \mathrm{g}, \mathrm{h}}$ \\ Udo Ehrmann $^{i}$ Tobias Franiel ${ }^{b, j, k}$ Thomas-Oliver Henkel ${ }^{a, b}, l$ Stefan Höcht ${ }^{e, m}$ \\ Glen Kristiansen $^{n, o, p}$ Stefan Machtens ${ }^{a, b}$, q Peter Niehoffr, s Tobias Penzkofer ${ }^{b, t}$ \\ Michael Pinkawa ${ }^{r, u} \quad$ Jan Philipp Radtke ${ }^{b, v}$ Wilfried Roth ${ }^{\text {n, o, w }}$ Ullrich Witzsch $^{\text {b, } x}$ \\ Roman Ganzer ${ }^{\mathrm{a}, \mathrm{y}} \quad$ Heinz Peter Schlemmerj, ${ }^{\mathrm{j}}$ Marc-Oliver Grimm ${ }^{\mathrm{a}, \mathrm{A}}$ Oliver W. Hakenberg ${ }^{\mathrm{a}, \mathrm{B}}$ \\ Martin Schostak ${ }^{a, b}, \mathrm{~h}$

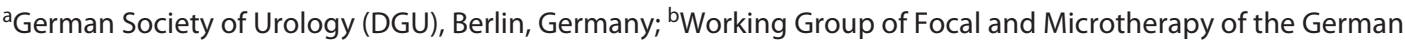 \\ Society of Urology (DGU), Berlin, Germany; ' Department of Urology, University Hospital, Technische Universität \\ Dresden, Dresden, Germany; dDepartment of Urology, Fuerth Hospital, Fuerth, Germany; eWorking Group \\ Radiooncology German Cancer Society (ARO), Berlin, Germany; ${ }^{f}$ Department of Radiooncology, University Hospital, \\ Charite Berlin, Berlin, Germany; 9ProUro, Berlin, Germany; hDepartment of Urology, University Hospital Magdeburg,

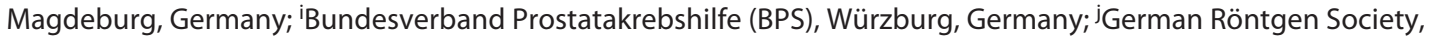 \\ Berlin, Germany; ${ }^{k}$ Department of Diagnostic and Interventional Radiology, University Hospital Jena, Jena, Germany;

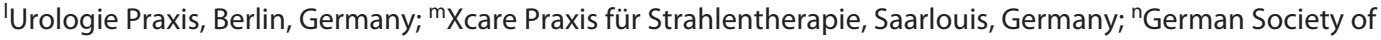 \\ Pathology, Berlin, Germany; ${ }^{\circ}$ Bundesverband Deutscher Urologen, Berlin, Germany; ${ }^{P}$ Department of Pathology, \\ Universitätsklinikum Bonn, Bonn, Germany; 'Department of Urology, GFO Hospitals Rhein-Berg, Marien-Hospital, \\ Bergisch-Gladbach, Germany; 'Berufsverband Deutscher Strahlentherapeuten, Berlin, Germany; ${ }^{\text {DDepartment of }}$ \\ Radiooncology, Sana Hospital Offenbach, Offenbach, Germany; tDepartment of Radiology, University Hospital \\ Charite Berlin, Berlin, Germany; "Department of Radiooncology, MediClin Robert Janker Hospital, Bonn, Germany;

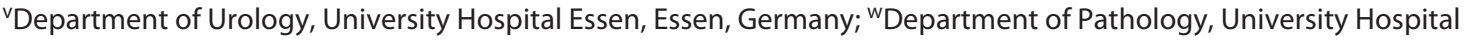 \\ Mainz, Mainz, Germany; 'Department of Urology, Krankenhaus Nordwest, Frankfurt, Germany; ' Department of \\ Urology, Asklepios Hospital Bad Tölz, Bad Tölz, Germany; ${ }^{2}$ Department of Radiology, German Cancer Research

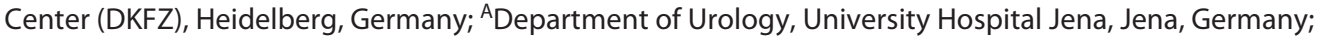 \\ ${ }^{B}$ Department of Urology, University Hospital Rostock, Rostock, Germany
}

\section{Keywords}

Evidence · Cryotherapy · Focal therapy - Guidelines . High-intensity focused ultrasound ablation - Prostate cancer · Irreversible electroporation · Vascular targeted photodynamic therapy

\footnotetext{
Abstract

Background: Focal therapy (FT) is an option to treat localized prostate cancer ( $\mathrm{PCa}$ ) and preserve healthy prostate tis-
}

sue in order to reduce known side effects from primary whole-gland treatment. The available FT modalities are manifold. Until now, national and international PCa guidelines have been cautious to propose recommendations regarding FT treatment since data from prospective controlled trials are lacking for most FT modalities. Moreover, none of the international guidelines provides a separate section on FT. In this purpose, we provide a synopsis of the consensusbased German S3 guidelines for a possible international use. Summary: The recently published update of the German S3 karger@karger.com www.karger.com/uin

Karger $\stackrel{\text { ' }}{5}$

GOPEN ACCESS
(C) 2022 The Author(s)

Published by S. Karger AG, Basel

This is an Open Access article licensed under the Creative Commons Attribution-NonCommercial-4.0 International License (CC BY-NC) (http://www.karger.com/Services/OpenAccessLicense), applicable to the online version of the article only. Usage and distribution for commercial purposes requires written permission.
Correspondence to:

Angelika Borkowetz, angelika.borkowetz@uniklinikum-dresden.de 
guidelines, an evidence- and consensus-based guideline, provides a section on FT with recommendations for diagnostic work-up, indications, modalities, and follow-up. This section consists of 12 statements and recommendations for FT in the treatment of localized PCa. Key Message: The German S3 guidelines on PCa are the first to incorporate recommendations for FT based on evidence and expert consensus including indication criteria for FT, pretreatment, and followup diagnostic pathways as well as an extended overview of FT techniques and the current supportive evidence.

(C) 2022 The Author(s).

Published by S. Karger AG, Basel

\section{Introduction}

Focal therapy (FT) is an option for treatment of localized prostate cancer $(\mathrm{PCa})$ with preservation of healthy prostate tissue with the aim of reducing known side effects of primary whole-gland treatments such as radical prostatectomy (RP) or radiotherapy $[1,2]$. Hypothetically, the advantages of FT are a higher rate of preserving both sexual and bladder function while simultaneously providing sufficient oncological control $[1,2]$. The possible FT modalities are manifold. To date, data from prospective controlled trials for most of the offered modalities are lacking. However, many retrospective studies have been published for most of the established FT modalities. So far, available prospective comparative data have shown neither a benefit in survival nor a lower complication profile of FT in comparison to RP or radiotherapy $[3,4]$. Therefore, national and international guidelines on PCa have been reluctant to provide recommendations for FT [5-9]. Currently, there are neither international nor national guidelines, which have a dedicated section on FT. The European Association of Urology (EAU) published a position paper in 2018, based on the current literature, providing a summary on FT modalities, requirements for patient recruitment and for follow-up [2].

The German S3 guideline on PCa [10] is an interdisciplinary, evidence- and consensus-based guideline issued by the Guideline Program Oncology of the Working Group of Medical Societies, the German Cancer Association (DKG), and the German Cancer Aid (DKH). In its most current update, the German S3 guidelines provide a dedicated section proposing recommendations on indications, requirements, and utilization of FT modalities for the treatment of localized and locally advanced PCa. This work aims to give an overview of these evidencebased FT guidelines for a potential international use.
Table 1. Expression of consensus power during structural consensus meeting

\begin{tabular}{ll}
\hline Consensus power & Consent, \% \\
\hline Strong consent & $>95 \%$ of mandates \\
Consent & $>75-95 \%$ of mandates \\
Major consent & $50-75 \%$ of mandates \\
Dissent & $<50 \%$ of mandates \\
\hline
\end{tabular}

\section{Description of Methods}

The German S3 guidelines on PCa were first published in 2009 with an update every $2-3$ years. They provide evidence- and consensus-based recommendations for early detection, diagnosis, and therapy of PCa in all tumor stages to improve the health care of patients with PCa. The guidelines are aimed at affected men, as well as professionals treating PCa patients. A systematic literature research and evaluation performed by the Medical Center for Quality in Medicine on behalf of the German Society of Urology (DGU) was conducted prior to consensus meetings to search for adequate published data.

Guidelines classified as S3 are methodologically based on a systematic review and synthesis of evidence combined with a structured process of consensus guided by a representative committee [11]. The development of these guidelines is based on the guidance manual and rules for guideline development [11].

Briefly, a structured systematic literature research was performed for aggregated evidence and randomized controlled studies using the data bases Medline via Pubmed and the Cochrane database. Additional publications were found by scrutinizing the reference lists of the identified studies. Only studies involving $>50$ patients were included for further evidence synthesis. The literature research was performed on August 25, 2020. Identified publications were screened by title and abstract as well as by full-text screening. Synthesis of evidence was performed by the Medical Center for Quality in Medicine. The systematic reviews and randomized controlled trials included were evaluated according to the AMSTAR2tool and to the Cochrane risk of bias tool, respectively [10]. All included studies and systematic reviews are depicted in evidence tables published in the guideline report [12].

The bias risk and the graduation of evidence of the identified studies were evaluated according to the Scottish Intercollegiate Guidelines Network [13]. According 
Table 2. Graduation of recommendation [11]

\begin{tabular}{lll}
\hline $\begin{array}{l}\text { Grade of } \\
\text { recommendation }\end{array}$ & Characterization & Used expression \\
\hline A & Strong recommendation & Should/should not (German "soll") \\
B & Recommendation & Ought to/ought not to (German "sollte") \\
0 & Open recommendation & May be considered/no specific recommendation \\
\hline
\end{tabular}

to the Guideline Program Oncology, the grade of recommendation is based on a consensus process by the authors of these guidelines. The process consists of nominal group processes with a certain number of experts on one topic/ section, as well as a structured consensus conference, both moderated by the Guideline Program Oncology of the Working Group of Medical Societies. In these processes, members of different medical societies and patient group representatives are involved. Sixty-nine interdisciplinary mandates are permitted to vote on the recommendations elaborated by the expert working groups. The results of the voting for every recommendation are expressed by the consensus power depicted in Table 1 .

For all evidence-based statements and recommendations, the level of evidence of the identified studies and the grade of recommendation, if available, are indicated. Three different levels of recommendation classify its strength, which is reflected by the expression used in the recommendation (Table 2). Next, statements are defined as declarations and explanations of specific issues without direct order. They are passed by formal consensus finding and are based either on study results or expert opinions. Statements and recommendations based on consented expert opinion are indicated as expert consensus (EC).

All members of the expert groups and all mandates had to declare any conflicts of interest in advance. In case of conflict of interest interfering with a vote on a recommendation/statement, the expert/mandate was advised not to vote for this statement/recommendation. The declarations on conflicts of interests of all members are published in the guidelines report [14].

The FT section of the guidelines was elaborated by a team of 18 German experts in FT and brachytherapy (urologists, radio-oncologists, radiologist, and pathologist) on study results or expert opinions. In this work, the recommendations and statements of the guidelines are depicted with adapted extraction of the background text to every recommendation and statement. The extractions were chosen by members of the expert group. The recommendations are indicated in italic.

German Guidelines on Focal Therapy

\section{Guidelines Body}

The section on FT of the German S3 guidelines consists of 13 recommendation statements. Twelve are given in the section on treatment of localized PCa (Table 3). One recommendation pertains to treatment of locally advanced PCa (not shown here). The FT section is divided into (i) general recommendations including diagnostics and follow-up and (ii) specific recommendations regarding FT modalities.

\section{General Recommendations on Inclusion Criteria, Diagnostic Steps, and Follow-Up}

The expert committee states that FT treats only a part of the prostate (consensus-based statement, EC, overall agreement: $91 \%$ ). In principle, FT of localized PCa is a minimally invasive procedure following the therapeutic concept of partial-gland treatment to treat only the region affected by cancer and a safety margin. Therefore, one tumor site or several closely located PCa foci are treated. FT has a curative goal. Multifocality and disease heterogeneity are limitations to the concept of partial or focal gland therapy. Success of FT is ultimately related to the accuracy of diagnosis and identification of tumor sites within the prostate.

The aim of FT in localized PCa is the eradication of all significant tumor tissue (consensus-based statement, EC, overall agreement: $86 \%$ ). This statement implies that FT intends to target and effectively treat all significant tumor foci within the gland. To achieve this, the region affected by PCa must be exactly localized and defined in order to be accessible for a FT modality with curative intention. Therefore, the authors defined tumor characteristics suitable for FT.

Due to the lack of data on the use of FT in both locally advanced and localized intermediate-risk PCa, the guidelines recommend that the use of FT is limited to patients with unilateral, localized low-risk PCa if they refuse both 


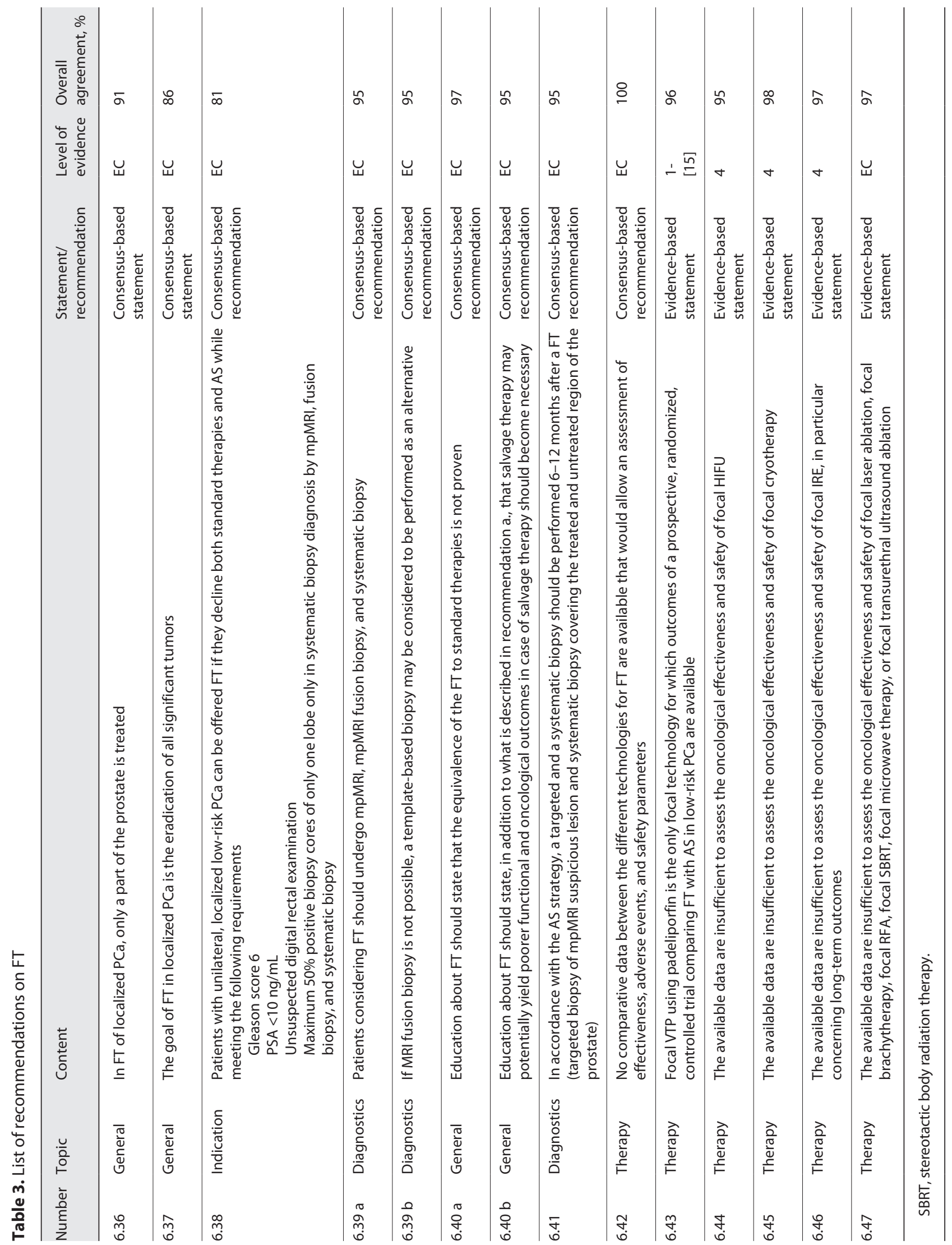


standard therapies and active surveillance (AS). Criteria for this recommendation include a Gleason score $3+3$; PSA $<10 \mathrm{ng} / \mathrm{mL}$; unsuspicious digital rectal examination and a maximum of $50 \%$ positive biopsy cores of one lobe only in systematic biopsy cores. The diagnosis is based on mpMRI, fusion biopsy, and systematic biopsy (consensus-based recommendation, EC, overall agreement: $81 \%)$.

Especially, the latter is important to identify all significant PCa foci. Therefore, patients considering FT should undergo mpMRI, mpMRI fusion biopsy, and mandatorily accompanied by systematic biopsy (consensus-based recommendation, EC, overall agreement: 95\%). If MRI fusion biopsy is not possible, a template-based biopsy may be considered to be performed as an alternative (consensus-based recommendation, EC, overall agreement: 95\%).

Since comparative data for FT and standard treatment are missing, guidelines pointed out that the patient's education about FT should state that the equivalence of FT to standard therapies is not proven (consensus-based recommendation, EC, overall agreement: 97\%). Moreover, patients should be aware that salvage therapy following FT may potentially yield poorer functional and oncological outcomes (consensus-based recommendation, EC, overall agreement: 98\%).

Follow-up of FT should include an mpMRI specifically assessing the treated area, but also systematically assessing the remaining untreated prostate followed by a combination of systematic and targeted control biopsy. This is necessary to determine the success of local treatment and detect or exclude possible heterotopic cancer foci. In accordance with the AS strategy, a targeted and a systematic biopsy should be performed 6-12 months after FT (targeted biopsy of mpMRI suspicious lesion and systematic biopsy covering the treated and untreated region of the prostate) (consensus-based recommendation, EC, overall agreement: 95\%). Detection of significant PCa in the treated area after 6-12 months indicates treatment failure of FT.

\section{Statements and Recommendations on FT Modalities}

The large number of FT modalities offered makes it difficult to assess the various individual procedures. This is complicated by the fact that the available data for each individual FT are insufficient. Moreover, in various publications, authors have not systematically differentiated between partial-gland and whole-gland treatment, pri- mary and salvage therapy, and localized low-risk and locally advanced PCa. In addition, cohorts are often enhanced by patients suffering from intermediate- and partially high-risk PCa. This leads to an indifferent guideline statement that no comparative data between the different technologies for FT exist, which allow an assessment of effectiveness, adverse events, and safety parameters (consensus-based statement, EC, overall agreement: 100\%). The recommendations of the guideline panel intend to ensure that individual FT modalities are only performed according to clear indications and strictly controlled by follow-up biopsy.

\section{Vascular Targeted Photodynamic Therapy}

The guidelines state that focal vascular targeted photodynamic therapy (VTP) using padeliporfin is the only FT technology for which outcomes of a prospective, randomized, controlled trial comparing FT with AS in low-risk PCa are available (evidence-based statement, level of evidence 1-, overall agreement: 96\%) [15]. In VTP, laser light of a wavelength of $753 \mathrm{~nm}$ activates the photosensitizing substance padeliporfin in the presence of oxygen, resulting in local tissue necrosis. Padeliporfin is administered intravenously and the prostate is, then, immediately treated for $22 \mathrm{~min}$ via optical laser fibers previously inserted transperineally into the area to be treated [15].

VTP has been approved by the European Medicine Agency for the treatment of untreated localized low-risk PCa (inclusion criteria: cT1c or cT2a, Gleason score $3+$ 3 , PSA $<10 \mathrm{ng} / \mathrm{mL}$, max. three positive biopsy cores with max. $5 \mathrm{~mm}$ in length of tumor infiltration for each of the three cores or 1-2 positive cores with $>50 \%$ tumor infiltration or a PSA density $\geq 0.15 \mathrm{ng} / \mathrm{mL} / \mathrm{mm}^{3}$ ). The approval was based on a prospective, randomized, openlabel, multicenter Phase III trial conducted in Europe, comparing VTP with AS in 413 patients with unilateral low-risk PCa [15]. The primary endpoint "negative biopsy after 12 months" was achieved in $48 \%$ of the VTP group and $20 \%$ of the AS group, with an odds ratio of 3.67 in favor of VTP $(p<0.001)$ [15]. The 4 -year outcomes of this study were reported for $266 / 413$ patients with the endpoint "conversion to radical therapy" [16]. The conversion rate in the VTP group compared to the AS group was $7 \%$ versus $32 \%$ after 2 years, $15 \%$ versus $44 \%$ after 3 years, and $24 \%$ versus $53 \%$ after 4 years ( $p<0.001$ each) [16]. 


\section{High-Intensity Focused Ultrasound Ablation}

Concerning high-intensity focused ultrasound ablation (HIFU), guidelines state that the available data are insufficient in order to assess the oncological effectiveness and safety of HIFU (evidence-based statement, level of evidence 4, overall agreement: 95\%). In HIFU, highenergy sound waves converging in a single point are focused on the prostate via an ultrasound probe placed in the rectum. The ultrasound beam causes vibrations in the tissue at the focal point. This results in a mechanical cavitation effect and a thermal effect with generation of heat. The energy source is a piezoelectric transducer which generates ultrasonic waves of varying frequency depending on the voltage. The absorption of ultrasonic ultrasound energy causes the temperature to rise to $75-90^{\circ} \mathrm{C}$ after $1 \mathrm{~s}$ of application and the formation of elliptically configured coagulative necrosis zones $50-300 \mathrm{~mm}^{2}$ in size. Larger treatment zones are generated by combining individual applications.

Case series, systematic reviews of case series, and one study on the comparison with cryotherapy were identified for HIFU. A systematic review without qualitative assessment of included studies and without meta-analysis analyzed 13 studies and a total of 543 patients [17]. The biochemical recurrence-free survival reported in only two studies was $88 \%$ [18] and $83 \%$ after 2 years for lowrisk PCa and 54\% [19] for intermediate-risk PCa [17]. Another review evaluated 16 series with at least 100 patients and a total number of 5,094 men [20]. Out of these, disease-free survival was reported in 11 studies, predominantly using PSA levels according to the PHOENIX criteria of ASTRO varying between $53 \%$ after 3 years and $97 \%$ after 10 years [20].

\section{Focal Cryotherapy}

The available data are insufficient to assess the oncological effectiveness and safety of focal cryotherapy (evidence-based statement, level of evidence 4 , overall agreement: 98\%). The goal of local cryotherapy is curative focal treatment with destruction of the localized carcinoma cancer site(s) by means of tissue necrosis generated by localized cooling to $-40^{\circ} \mathrm{C}$. The cryoneedles are TRUScontrolled and inserted into the prostate via a perineal template; additional thermosensor needles continuously register the temperature in the vicinity.

In a randomized controlled trial comparing cryotherapy and radiotherapy in a total of 244 patients [21], no differences in overall or disease-specific survival were detected. The positive biopsy rate at 36 months was $29 \%$ in the radiotherapy group and $7.7 \%$ in the cryoablation group [21]. A Cochrane review of cryotherapy as the primary therapy identified 8 cohort studies including a total number of 1,483 patients [22]. Four of the eight studies showed a positive follow-up biopsy in $17-28 \%$ of patients [22]. The other four studies reported progression-free survival in $71-89 \%$ and positive follow-up biopsies in $1.4-13 \%$ of patients [22]. Overall survival after 5 years was $89-92 \%$ (two studies), and the disease-specific survival was $94 \%$ in one study [22]. Another meta-analysis of controlled trials comparing cryotherapy with radiotherapy and cryotherapy with RP, as well as single-arm studies reporting cryotherapy outcomes, identified 10 publications from seven trials with a total number of 1,252 patients [23]. There were no significant differences between cryotherapy and radiotherapy plus RP regarding to overall survival and tumor-specific survival, but a significantly lower biochemical recurrence-free survival after cryotherapy [23]. However, it must be taken into consideration that in all of these studies, cryotherapy was performed as a whole-gland ablation therapy. Data for focal cryotherapy are rare. Therefore, the recommendation only attained a level of evidence grade 4 .

\section{Focal Irreversible Electroporation}

The available data are insufficient to assess the oncological effectiveness and safety of focal irreversible electroporation (IRE), in particular, concerning long-term outcomes (evidence-based statement, level of evidence 4, overall agreement: $97 \%$ ).

Electroporation means that tissue necroses are caused by the current flowing between two electrodes inserted into the prostate. This necrosis is not the result of generated heat but is caused by disruption of cell membranes. It is postulated that better sparing of sensitive structures (urethra, neurovascular bundles) can be achieved compared to other ablative methods based on a change in temperature [24].

To date, very little data are available for this ablative technique when applied to focal PCa. In a prospective cohort study, 30 patients with low-risk or intermediate-risk PCa were investigated. In 28 of 30 patients, a biopsy was performed 6 months after the intervention. Evidence of persistent PCa in the field of treatment was found in $5 / 28$ (18\%) [25]. A prospective registry study investigated 12-month data (median follow-up 36 months) for 123 patients with IRE (91\% intermediate-risk PCa, 9\% low-risk $\mathrm{PCa}$ ) [25]. Tumor persistence in the treated area was con- 
sidered in $2.7-9.8 \%$ at 12 months [25]. A systematic review identified 10 publications with a total of 433 patients [26]. 265/433 patients had an intermediate-risk PCa with a follow-up of 6-36 months [26]. The rate of PCa persistence in the treated area was $0-39 \%$, and the rate of newly diagnosed PCa outside the treated area was $6.4-24 \%$ [26].

\section{Other FT Approaches}

The available data are insufficient to assess the oncological effectiveness and safety of focal laser ablation, focal brachytherapy, focal radiofrequency ablation (RFA), focal stereotactic body radiation therapy, focal microwave therapy, or focal transurethral ultrasound ablation (consensus-based statement, EC, overall agreement: $100 \%)$. There is no evidence regarding effectiveness and no safety data available for these procedures. Therefore, none of the following procedures: focal brachytherapy, focal RFA, focal stereotactic radiation therapy, and focal microwave therapy are recommended for general patient treatment. Their use is therefore restricted to prospective, controlled trials.

Herein, we present the first evidence-based S3 guidelines on PCa with a detailed chapter on FT. Within this chapter, the diagnostic workflow before FT, the inclusion criteria and follow-up are elaborated. Moreover, individual important FT techniques are explained in detail, and attention is drawn to the most important supporting evidence. In contrast, other national and international guidelines mention HIFU or cryotherapy as treatment options mainly as whole-gland treatment and regard these treatment options as experimental therapy options which should be offered only within clinical trials. The guidelines of the Belgian Society of Urology, published in 2013, are the only guidelines, which have a detailed chapter on the evidence for HIFU [7]. In total, 12 relevant systematic reviews and 23 primary studies were identified by the authors [7]. However, these guidelines consider HIFU as a treatment option in men with localized PCa only in the context of controlled clinical trials with a low level of evidence and a weak strength of recommendation. In addition, there is no recommendation for cryotherapy in these guidelines [7]. Furthermore, it should be noted that the last update was in 2013. In addition, the National Comprehensive Cancer Network guidelines, last updated in February 2021, do not mention any FT in localized PCa. Whole-gland HIFU is considered as a salvage therapy option in the case of PCa recurrence after radiotherapy [8]. The National Institute for Health and Care Excellence guidelines recommend HIFU and cryotherapy only to men with localized PCa in the context of controlled clinical trials comparing their use with established interventions. However, again, this recommendation was last updated in 2008 [5]. The American Association of Urology guideline, most recent update in 2017, mentions only cryotherapy as whole-gland ablation and HIFU as FT modality. HIFU is recommended only as a FT treatment option lacking robust evidence for efficacy. Even though HIFU is approved by the FDA, patients should be advised that it is not approved explicitly for the treatment of PCa. Moreover, HIFU has a moderate recommendation and Grade $\mathrm{C}$ level of evidence in terms of the tumor location possibly influencing the oncological outcome in HIFU therapy. Additionally, they point out the risk of multifocality of PCa. Therefore, clinicians are advised to inform patients that FT may not be curative, and that, further treatment for PCa may be necessary [6].

The guidelines of the EAU recommend FT only within the context of a clinical trial setting or within a welldesigned prospective cohort study due to the lack of robust comparative data on medium- to long-term oncological outcomes [9]. However, recommendations for pre-FT diagnostic evaluation or follow-up are missing. In 2018, the European Association published a position paper on FT in localized PCa [2]. They reported on several consensus reports for the diagnostic evaluation before and indication for FT in PCa. Moreover, they summarized the main FT modalities based on the current literature. However, they did not perform a systematic literature search. They commented on the main questions concerning the aim of FT. Herein, they considered the restriction of image modalities to rule out high-risk PCa in the pretreatment setting and the lack of follow-up after FT. Moreover, reliable comparative data for most FT modalities compared to standard treatment or AS are still missing [2]. The authors of this position paper state that FT should be considered as an investigational modality only.

In contrast to the previous mentioned data and guideline recommendations, all recommendations stated in the novel German S3 guideline are based on consensusbased expert recommendation. We would like to point out that these guidelines are the first defining the indication criteria for patients eligible for FT and can be offered to patients with unilateral, localized low-risk PCa; only however, if they refuse both standard therapies and AS 
while meeting the following requirements: Gleason score 6 , PSA $<10 \mathrm{ng} / \mathrm{mL}$, and nonsuspicious DRU with a maximum of $50 \%$ of the biopsy cores positive in one lobe. The diagnosis should be based on mpMRI, fusion biopsy, and systematic biopsy. The FT modalities VTP, focal HIFU, focal cryotherapy, and IRE are described in detail in subchapters. However, only VTP is the sole FT identified as evidence-based statement with Level 1-evidence, since the only prospective randomized controlled trial for a FT modality compared VTP with AS in patients with unilateral low-risk PCa.

The guidelines recommend FT only in case of unilateral low-risk PCa which can be offered in patients refusing AS in unilateral low-risk PCa. Therefore, this version of the guidelines mentions VTP treatment only as evidence-based statement with a level of evidence 1 and does not recommend VTP as primary treatment option in patients with unilateral low-risk PCa. Retrospective evaluation of VTP [27] underlines the critical consideration of this method in daily practice.

The EAU guideline highlighted this as well. In the novel German S3 guidelines, focal HIFU, cryotherapy, and IRE are mentioned as evidence-based statements with an evidence level of 4 due to insufficient data assessing the oncological outcome and safety. In contrast, available data for other FT modalities, such as focal brachytherapy, RFA, or laser therapy are insufficient regarding the oncological outcome and safety. All recommendations and statements of the German S3 guidelines are defined cautiously due to the lack of robust data for the most mentioned issues. This is reflected in the number of statements based on EC. Moreover, this guideline presents technical considerations and the most important evidence of every single FT modality. Based on data for the primary diagnosis of $\mathrm{PCa}$, these guidelines provide recommendations on pretreatment and follow-up diagnosis, reflecting the prudent management of FT modalities. This is underlined especially by the statement that patients should be informed about the possibility of worse functional outcome and higher rate of positive surgical margins in case of salvage treatment. Due to the statement on the abovementioned restricted indication of FT based on EC, the guidelines do not regard FT as a solely experimental therapy option. However, data from ongoing, prospective trials are necessary to upgrade the evidence of FT in PCa therapy. As there is an obvious need to explore the use of FT for patients with intermediate-risk localized $\mathrm{PCa}$, future studies should concentrate on patients with these risk features. However, for FT modalities, e.g., laser ablation or focal RFA, for which scarce data from unicentric and retrospective studies with only a small number of patients exist $[28,29]$, these guidelines recommend their use only in prospective, controlled trials. Since conducting controlled trials comparing new FT modalities with standard treatments such as RP or radiotherapy is difficult, their usage should be restricted to clinical trials. It would also make sense to develop controlled study concepts comparing whole and partial prostate gland treatments for one and the same FT modality. These studies could deliver information on the effect of a specific treatment modality either applied as a wholegland or focal approach.

\section{Conclusion}

The German S3 PCa guidelines are the first guidelines with recommendations for FT based on evidence or EC, which include indications for FT, pretreatment, and follow-up diagnostic pathways as well as an extended overview of FT techniques and their current evidence. Due to the lack of robust data on long-term oncological outcomes, these guidelines should be regarded as "guidelines in progress" and be considered in further updates.

\section{Acknowledgments}

We would like to thank the Agency for Quality in Medicine Joint Institution of the German Medical Association and the $\mathrm{Na}$ tional Association of the Statutory Health Insurance Physicians, especially Katrin Krueger and Corinna Schaefer, for providing methodological and structural support in the development of the guideline. We thank Philip Mackenzie for proofreading.

\section{Conflict of Interest Statement}

The authors have no conflicts of interest to declare.

\section{Funding Sources}

This research did not receive any specific grant from funding agencies in the public, commercial, or not-for-profit sector.

\section{Author Contributions}

The followings are the authors' contributions: A.Bo.: project development, guidelines development, and manuscript editing; A.Bl.: guidelines development and manuscript editing; D.B.: 
guidelines development and manuscript editing; H.C.: guidelines development and manuscript editing; U.E.: guidelines development; T.F.: guidelines development; T.-O.H.: guidelines development; S.H.: guidelines development and manuscript editing; G.K.: guidelines development; S.M.: guidelines development and manuscript editing; P.N.: guidelines development; T.P.: guidelines development and manuscript editing; M.P.: guidelines development and manuscript editing, J.P.R.: guidelines development and manuscript editing; W.R.: guidelines development; U.W.: guidelines development and manuscript editing; R.G.: guidelines development, manuscript editing; H.P.S.: guidelines development; M.-O.G.: guidelines development and manuscript editing; O.W.H.: guidelines development and manuscript editing; and M.S.: project development, guidelines development, and manuscript editing.

\section{References}

1 Valerio M, Cerantola Y, Eggener SE, Lepor H, Polascik TJ, Villers A, et al. New and established technology in focal ablation of the prostate: a systematic review. Eur Urol. 2017; 71(1):17-34.

2 van der Poel HG, van den Bergh RCN, Briers E, Cornford P, Govorov A, Henry AM, et al. Focal therapy in primary localised prostate cancer: the European Association of Urology Position in 2018. Eur Urol. 2018;74(1):84-91.

3 Jin K, Qiu S, Zheng X, Li Y, Zhang S, Li J, et al. Cryotherapy shows no inferiority compared with radical prostatectomy for low-risk and intermediate-risk localized prostate cancer: a real-world study from the SEER database. J Cancer. 2020;11(19):5738-45.

4 Jung JH, Risk MC, Goldfarb R, Reddy B, Coles B, Dahm P. Primary cryotherapy for localised or locally advanced prostate cancer. Cochrane Database Syst Rev. 2018;5:CD005010.

5 Excellence NIfHaC. Prostate cancer: diagnosis and management. 2019.

6 Available from: https://www.auanet.org/ guidelines/guidelines/prostate-cancer-clinically-localized-guideline. 2017.

7 Mambourg FJP, Piérart J, Van Brabandt H. A national clinical practice guideline on the management of localised prostate cancer. Good clinical practice (GCP). Brussels: Belgian Health Care Knowledge Centre (KCE); 2012. KCE reports 194C. D/2012/10.273/101. 2012.

8 Mohler JL, Antonarakis ES, Armstrong AJ, D'Amico AV, Davis BJ, Dorff T, et al. Prostate cancer, version 2.2019, NCCN clinical practice guidelines in oncology. J Natl Compr Canc Netw. 2019;17(5):479-505.

9 Mottet N, van den Bergh RCN, Briers E, Van den Broeck T, Cumberbatch MG, De Santis $\mathrm{M}$, et al. EAU-EANM-ESTRO-ESUR-SIOG guidelines on prostate cancer-2020 update. Part 1: screening, diagnosis, and local treatment with curative intent. Eur Urol. 2021; 79(2):243-62.

10 Leitlinienprogramm onkologie (Deutsche Krebsgesellschaft DK, AWMF): S3-leitlinie prostatakarzinom L, 2021, AWMF registernummer: 043/022OL hwl-odlp.
11 German Association of the Scientific Medical Societies (AWMF) - Standing Guidelines Commission. AWMF guidance manual and rules for guideline development. 1st ed. 2012. English version.

12 Leitlinienprogramm onkologie (Deutsche Krebsgesellschaft DK, AWMF): S3-leitlinie prostatakarzinom, evidenzbericht, version 6.0, Mai 2021, AWMF registernummer: 043/022OL. Available from: http://leitlinienprogramm-onkologie.de/Prostatakarzinom.58.0.html 2021.

13 Available from: www.sign.ac.uk/pdf/sign50. pdf.

14 Leitlinienprogramm onkologie (Deutsche Krebsgesellschaft DK, AWMF): S3-leitlinie prostatakarzinom, leitlinienreport, version 6.0, Mai 2021, AWMF registernummer: 043/022OL. Available from: https://www. leitlinienprogramm-onkolo-gie.de/leitlinien/ prostatakarzinom/2021.

15 Azzouzi AR, Emberton M; PCM301 study investigators. Padeliporfin vascular-targeted photodynamic therapy versus active surveillance in men with low-risk prostate cancer authors' reply. Lancet Oncol. 2017;18(4): e188.

16 Gill IS, Azzouzi AR, Emberton M, Coleman JA, Coeytaux E, Scherz A, et al. Randomized trial of partial gland ablation with vascular targeted phototherapy versus active surveillance for low risk prostate cancer: extended followup and analyses of effectiveness. J Urol. 2018;200(4):786-93.

17 Golan R, Bernstein AN, McClure TD, Sedrakyan A, Patel NA, Parekh DJ, et al. Partial gland treatment of prostate cancer using high-intensity focused ultrasound in the primary and salvage settings: a systematic review. J Urol. 2017;198(5):1000-9.

18 Shoji S, Nakano M, Fujikawa H, Endo K, Hashimoto A, Tomonaga T, et al. Urethrasparing high-intensity focused ultrasound for localized prostate cancer: functional and oncological outcomes. Int J Urol. 2015;22(11): 1043-9.

19 Muto S, Yoshii T, Saito K, Kamiyama Y, Ide $\mathrm{H}$, Horie S. Focal therapy with high-intensityfocused ultrasound in the treatment of localized prostate cancer. Jpn J Clin Oncol. 2008; 38(3):192-9.
20 Ziglioli F, Baciarello M, Maspero G, Bellini V, Bocchialini T, Cavalieri D, et al. Oncologic outcome, side effects and comorbidity of high-intensity focused ultrasound (HIFU) for localized prostate cancer. A review. Ann Med Surg. 2020;56:110-5.

21 Donnelly BJ, Saliken JC, Brasher PM, Ernst $\mathrm{SD}$, Rewcastle JC, Lau H, et al. A randomized trial of external beam radiotherapy versus cryoablation in patients with localized prostate cancer. Cancer. 2010;116(2):323-30.

22 Shelley M, Wilt TJ, Coles B, Mason MD. Cryotherapy for localised prostate cancer. Cochrane Database Syst Rev. 2007;3:CD005010.

23 Gao L, Yang L, Qian S, Tang Z, Qin F, Wei Q, et al. Cryosurgery would be an effective option for clinically localized prostate cancer: a meta-analysis and systematic review. Sci Rep. 2016;6:27490.

24 Blazevski A, Scheltema MJ, Amin A, Thompson JE, Lawrentschuk N, Stricker PD. Irreversible electroporation (IRE): a narrative review of the development of IRE from the laboratory to a prostate cancer treatment. BJU Int. 2020;125(3):369-78.

25 Collettini F, Enders J, Stephan C, Fischer T, Baur ADJ, Penzkofer T, et al. Image-guided irreversible electroporation of localized prostate cancer: functional and oncologic outcomes. Radiology. 2019;292(1):250-7.

26 Morozov A, Taratkin M, Barret E, Singla N, Bezrukov E, Chinenov D, et al. A systematic review of irreversible electroporation in localised prostate cancer treatment. Andrologia. 2020;52(10):e13789.

27 Flegar L, Buerk B, Proschmann R, Propping S, Groeben C, Baunacke M, et al. Vasculartargeted photodynamic therapy in unilateral low-risk prostate cancer in Germany: 2-yr single-centre experience in a real-world setting compared with radical prostatectomy. Eur Urol Focus. 2021. Epub ahead of print.

28 Lindner U, Lawrentschuk N, Trachtenberg J. Focal laser ablation for localized prostate cancer. J Endourol. 2010;24(5):791-7.

29 Orczyk C, Barratt D, Brew-Graves C, Peng Hu Y, Freeman A, McCartan N, et al. Prostate radiofrequency focal ablation (ProRAFT) trial: a prospective development study evaluating a bipolar radiofrequency device to treat prostate cancer. J Urol. 2021;205(4):1090-9. 